\title{
Remarks on thermodynamical terminology
}

\author{
Wolfgang Muschik \\ Institut für Theoretische Physik, Technische Universität Berlin, Germany \\ Communicated by J.U. Keller, Siegen, Germany
}

Registration Number 025

Often difficulties in understanding thermodynamics arise from using different terminology or semantics. Therefore here some few remarks are made for tending towards a unified thermodynamical terminology.

First of all one has to distinguish between two different description of thermodynamical systems: Such a system can be described as a discrete (or lumped) system or by field formulation. Discrete systems are separated from its environment by partitions. The interaction between the discrete system and its environment can be described by exchanges, namely by heat exchange, by power exchange and by material exchange (Schottky system) [1]. The discrete system itself is characterized by in general time-dependent quantities, the basic variables, belonging to the whole system [2]. This is of course a reduced model of a thermodynamical system. For a more detailed depiction of the system, it has to be described as a composite (or compound) system or by field formulation.

The exchanges between the system and the environment depend on both their basic variables. Because naturally no gradients appear in the description of discrete systems, this concept is used in thermostatics. But also complex machines in engineering sciences are described by discrete systems, if mainly exchange quantities are of interest. Thus, for a reduced description of complex situations, the concept of a discrete system is a very useful one.

As mentioned already, if more information is neccessary, we have to describe the thermodynamical system by field formulation. Here the quantities describing the system are in general $n$ time-dependent fields $\boldsymbol{a}(\boldsymbol{x}, t)$ [3] which satisfy the following balances

$$
\partial_{t}(\varrho \boldsymbol{a})+\nabla \cdot(\boldsymbol{v} \varrho \boldsymbol{a}+\boldsymbol{\Phi})=\boldsymbol{\Sigma}
$$

Here $\varrho(\boldsymbol{x}, t)$ is the field of the mass density, $\boldsymbol{v}(\boldsymbol{x}, t)$ the field of the material velocity, $\boldsymbol{\Phi}(\boldsymbol{x}, t)$ the fields of the conductive fluxes belonging to $\boldsymbol{a}$, and $\boldsymbol{\Sigma}(\boldsymbol{x}, t)$ the sum of the 
production and of the supply terms of $\boldsymbol{a}$. In general, not all of the a are wanted (or basic) fields. In non-extended thermodynamics [4], these basic fields are the following five ones

$$
(\varrho, \epsilon, \boldsymbol{v})(\boldsymbol{x}, t) .
$$

Here $\epsilon(\boldsymbol{x}, t)$ is the field of the internal energy.

The balance equations (1) are underdetermined: They represent $n$ equations for more than $n$ quantities. Consequently, we need additional equations for obtaining a determined system of differential equations. These additional equations are the constitutive equations which describe the considered material of which the system consists. For performing the derivatives in (1) we need a state space which is defined as the domain of the constitutive equations. In general the state space is different from the wanted fields [5]. In non-extended thermodynamics, it includes also derivatives of the wanted fields

$$
\boldsymbol{z}=\left(\varrho, \epsilon, \boldsymbol{v}, \nabla \varrho, \nabla \epsilon, \nabla \boldsymbol{v}, \partial_{t} \epsilon, \ldots\right)
$$

In extended thermodynamics [6,7], the state space does not contain derivatives of the basic fields, but instead of them the traceless stress tensor $\mathbf{P}^{0}$ and dissipative fluxes, such as the heat flux density $\boldsymbol{q}$

$$
\boldsymbol{z}=\left(\varrho, \epsilon, \boldsymbol{v}, \mathbf{P}^{0}, \boldsymbol{q}, \ldots\right)
$$

For these additional fields, one needs additional balances equations. The constitutive equations are now

$$
\boldsymbol{a}(\boldsymbol{z}), \boldsymbol{\Phi}(\boldsymbol{z}), \boldsymbol{\Sigma}(\boldsymbol{z})
$$

and the derivatives in (1) become

$$
\partial_{t} \boldsymbol{a}=\frac{\partial \boldsymbol{a}}{\partial \boldsymbol{z}} \cdot \partial_{t} \boldsymbol{z}, \quad \nabla \cdot \boldsymbol{a}=\frac{\partial \boldsymbol{a}}{\partial \boldsymbol{z}} \cdot \nabla \boldsymbol{z}
$$

If we now introduce (6) into the balances (1), we obtain by using the balance of mass

$$
\begin{aligned}
& \frac{\partial}{\partial t} \varrho(\boldsymbol{x}, t)+\nabla \cdot\{\varrho(\boldsymbol{x}, t) \boldsymbol{v}(\boldsymbol{x}, t)\}=0, \\
& \varrho \frac{\partial \boldsymbol{a}}{\partial \boldsymbol{z}} \cdot \partial_{t} \boldsymbol{z}+\varrho \boldsymbol{v} \frac{\partial \boldsymbol{a}}{\partial \boldsymbol{z}}: \nabla \boldsymbol{z}+\frac{\partial \boldsymbol{\Phi}}{\partial \boldsymbol{z}} \cdot \partial_{t} \boldsymbol{z}=\boldsymbol{\Sigma}(\boldsymbol{z}) .
\end{aligned}
$$

These balance equations are called balances on the state space. Dependent on the special constitutive equations this system of differential equations is highly nonlinear, but often, it is not sufficient to describe complex materials. For describing complex materials one need additional fields $\boldsymbol{b}(\boldsymbol{x}, t)$ beyond the $\boldsymbol{a}(\boldsymbol{x}, t)$. Additional fields demand additional equations for them

$$
\partial_{t}(\varrho \boldsymbol{b})+\nabla \cdot(\boldsymbol{v} \varrho \boldsymbol{b}+\boldsymbol{\Psi})=\boldsymbol{\Xi}
$$


and the original balances (1) contain different constitutive equations

$$
\partial_{t}(\varrho \boldsymbol{a})+\nabla \cdot\left(\boldsymbol{v} \varrho \boldsymbol{a}+\boldsymbol{\Phi}^{\prime}\right)=\boldsymbol{\Sigma}^{\prime} .
$$

The usual way to describe complex materials is to include additional fields and their governing equations (9). The difficulty which arises is the knowledge of these additional equations (9) and of the material equations $\boldsymbol{\Phi}^{\prime}(\boldsymbol{x}, t)$ and $\boldsymbol{\Sigma}^{\prime}(\boldsymbol{x}, t)$.

Another method of describing complex materials is the mesoscopic theory [8]. Instead of introducing additional fields, we introduce additional variables, called mesoscopic variables, which extend space-time

$$
(\boldsymbol{x}, t) \rightarrow(\boldsymbol{x}, t, \boldsymbol{b}) \equiv(\cdot) .
$$

The fields are now defined on the mesoscopic space (11):

$$
\varrho(\cdot), \boldsymbol{a}(\cdot), \boldsymbol{v}(\cdot), \boldsymbol{\Phi}(\cdot), \boldsymbol{\Sigma}(\cdot) .
$$

They are called the mesoscopic fields. Their balance equations are obtained by an additional derivation with respect to the mesoscopic variables $\boldsymbol{b}$

$$
\partial_{t}(\varrho \boldsymbol{a})(\cdot)+\nabla \cdot(\boldsymbol{v} \varrho \boldsymbol{a}+\boldsymbol{\Phi})(\cdot)+\nabla_{b} \cdot(\boldsymbol{u} \varrho \boldsymbol{a}+\boldsymbol{\Phi})(\cdot)=\boldsymbol{\Sigma}(\cdot) .
$$

Here $\boldsymbol{u}(\cdot)$ is the mesoscopic change velocity which is an analog to the material velocity and is defined as follows:

$$
(\boldsymbol{b}, \boldsymbol{x}, t) \rightarrow(\boldsymbol{b}+\boldsymbol{u}(\cdot) \Delta t, \boldsymbol{x}+\boldsymbol{v}(\cdot) \Delta t, t+\Delta t)
$$

The mesoscopic balance equations (13) have to be solved by methods which are also applied to the balances (1). Examples for mesoscopic variables are the dryness fraction in a two-phase flow, the microscopic director in liquid crystal theory [9], the damage parameter in crack theory [10] and the orientation of microcrystallites in microstructured media.

The mesoscopic theory introduces a statistical element, the mesoscopic distribution function [11]

$$
f(\cdot):=\frac{\varrho(\cdot)}{\int \varrho(\cdot) d \mathscr{M}}
$$

which represents the normalized mesoscopic mass density. Here the integration is performed on the mesoscopic variables of the mesoscopic space (11). Because of the definition (15) and of the mesoscopic mass balance

$$
\frac{\partial}{\partial t} \varrho(\cdot)+\nabla_{x} \cdot\{\varrho(\cdot) \boldsymbol{v}(\cdot)\}+\nabla_{b} \cdot\{\varrho(\cdot) \boldsymbol{u}(\cdot)\}=0,
$$

which results from (13) for $\mathrm{a} \equiv 1$, the mesoscopic distribution function satisfies the following balance equation 


$$
\begin{aligned}
& \frac{\partial}{\partial t} f(\cdot)+\nabla_{x} \cdot[\boldsymbol{v}(\cdot) f(\cdot)]+\nabla_{b} \cdot[\boldsymbol{u}(\cdot) f(\cdot)] \\
& \quad+f(\cdot)\left[\frac{\partial}{\partial t}+\boldsymbol{v}(\cdot) \cdot \nabla_{x}\right] \ln \varrho(\boldsymbol{x}, t)=0 .
\end{aligned}
$$

This mesoscopic balance equation contains the macroscopic mass density. Inserting it, we can see, that (18) is highly non-linear. The macroscopic influence on mesoscopic quantities is often denoted as a "mean field" theory.

The macroscopic fields are obtained by integrating over the mesoscopic variables [3]

$$
\begin{aligned}
& \varrho(\boldsymbol{x}, t)=\int \varrho(\cdot) d \mathscr{M}, \\
& v(\boldsymbol{x}, t)=\frac{1}{\varrho(\boldsymbol{x}, t)} \int \varrho(\cdot) \boldsymbol{v}(\cdot) d \mathscr{M}, \\
& \epsilon(\boldsymbol{x}, t)=\int\left\{\epsilon(\cdot)+\varrho(\cdot)[\boldsymbol{v}(\cdot)-\boldsymbol{v}(\boldsymbol{x}, t)]^{2}\right\} d \mathscr{M}, \ldots
\end{aligned}
$$

By the mesoscopic distribution function (15), we obtain a family of macroscopic order parameter fields which describe the complex material additionally to the usual fields (2)

$$
\begin{aligned}
& \int f(\cdot) d \mathscr{M}=1 \\
& \int \boldsymbol{b} f(\cdot) d \mathscr{M}=\boldsymbol{B}(x, t), \\
& \int \boldsymbol{b} \boldsymbol{b} f(\cdot) d \mathscr{M}=\mathscr{B}(\boldsymbol{x}, t), \\
& \int \boldsymbol{b} \ldots \text { mtimes } \ldots \boldsymbol{b} f(\cdot) d \mathscr{M}=\underline{\mathscr{B}}_{m}(\boldsymbol{x}, t) \text {. }
\end{aligned}
$$

Besides the balance equations (1) or (13), we have to take into consideration the macroscopic dissipation inequality by which the second law is introduced

$$
\partial_{t}(\varrho s)+\nabla \cdot(\boldsymbol{v} \varrho s+\phi)=\sigma \geq 0
$$

There are different possibilities of exploiting the dissipation inequality. The most simple one is first to solve the balances on the state space (8), and then to determine parameters in the constitutive equations in such a way that the dissipation inequality is satisfied. The second method of exploitation is those of Coleman and Noll [12]. This exploitation method does not take all balance equation into consideration as constraints, which is done by the Liu exploitation [13]. 
These few remarks may be sufficient for stimulating the discussion on thermodynamical terminology and on the concepts on which it is based. The original literature, where the concepts can be found, is cited below.

\section{References}

[1] Schottky, W., Thermodynamik, Erster Teil §1, Springer, Berlin, 1929.

[2] Muschik, W., Fundamentals of Non-Equilibrium Thermodynamics, in: Non-Equilibrium Thermodynamics with Application to Solids, 1-63, Chap. 1, Ed. Muschik, W., Springer, Wien, 1993.

[3] Muschik, W., Papenfuss, C., Ehrentraut, H., A sketch of continuum thermodynamics, J. Non-Newtonian Fluid Mech., 96 (2001), 255-290.

[4] Muschik, W., Aspects of Non-Equilibrium Thermodynamics, Sect. 6.3, World Scientific, Singapore, 1990.

[5] Muschik, W., Papenfuss, C., Ehrentraut, H., Concepts of Continuum Thermodynamics, Chap. 4, Kielce University of Technology, ISBN 83-905132-7-7, 1996.

[6] Jou, D., Casas-Vázquez, J., Lebon, G., Extended Irreversible Thermodynamics, Springer, Berlin, 1993.

[7] Müller, I., Ruggeri, T., Extended Thermodynamics, Springer, Berlin, 1993.

[8] Muschik, W., Ehrentraut, H., Papenfuss, C., Concepts of mesoscopic continuum physics with application to liquid crystals, J. Non-Equilib. Thermodyn. 25 (2000), 179-197.

[9] Muschik, W., Papenfuss, C., Ehrentraut, H., Mesoscopic theory of liquid crystals, J. Non-Equilib. Thermodyn. 29 (2004), 75-106.

[10] Papenfuss, C., Ván, P., Muschik, W., Mesoscopic theory of microcracks, Arch. Mech. 55 (2003), 481-499.

[11] Muschik, W., Papenfuss, C., Ehrentraut, H., Concepts of Continuum Thermodynamics, Chap. 5.4, Kielce University of Technology, ISBN 83-905132-7-7, 1996.

[12] Muschik, W., Aspects of Non-Equilibrium Thermodynamics, Sect. 6.4, World Scientific, Singapore, 1990.

[13] Muschik, W., Ehrentraut, H., An amendment to the second law, J. Non-Equilib. Thermodyn. 21 (1996), 175. 
Brought to you by | Technische Universität Berlin 